\title{
ALGUMAS CONSIDERAÇÕES SOBRE AVALIAÇÃO DO PACIENTE EM SALA DE RECUPERAÇÃO ANESTÉSICA
}

\section{PATIENT EVALUATION IN RECOVERY ROOM: SOME CONSIDERATIONS}

\author{
Aparecida de Cassia Giani Peniche*
}

PENICHE, A. de C.G. Algumas consideraç̃̃es sobre avaliação do paciente em sala de recuperação anestésica. Rev.Esc.Enf.USP, v.32, n.1, p. $27-32$, abr. 1998 .

\section{RESUMO}

A preocupą̧ão com a qualidade de assistência ao paciente tem sido uma constante ainda maior, quando relacionada a alta complexidade que envolve o cuidado do paciente em sala de recuperação anestésica (SRA). Acreditando ser necessário uma avaliação segura e eficaz, que considere também as alterações endócrinas e metabólicas decorrentes do trauma anestésico-cirúrgico do paciente, sugere-se a criação de padrões e critérios de avaliação para a assistência prestada ao paciente em SRA e a validação dos mesmos.

UNITERMOS: Recuperação anestésica. Avaliação do paciente.

\begin{abstract}
The quality in patient assistence concerne has been more constant than the high complexity of patient treatment needed in an anaesthetic recovery room. Thinking that is necessary to have assurance and efficiency in any evaluation, which has to consider all endocrine and metabolic variations resulting from patient surgical trauma, may be suggested the standardization and criterization of evaluation methods to patient assistence, and of course the validation of them.
\end{abstract}

UNITERMS: Anaesthetic evaluation. Patient evaluation .

Desde 1801 existe registro, na Inglaterra, de um local reservado, ao lado da sala de operações, aos pacientes em estado crítico ou recém submetidos à cirurgia de grande porte.

Apesar da existência dessa sala de observação pós-anestésica, como foi chamada no princípio, os objetivos específicos so foram delineados em 1944 (NOCITE, 1987; FRAULINI, 1987).

Atualmente, como citam vários autores (ELTRINGHAM; DURIIN; ANDREWES, 1986; DRAIN; SHIPLEY, 1981; FRAULINI, 1987) esses objetivos visam a centralização dos pacientes após o término do procedimento anestésico cirúrgico até que haja a estabilidade dos sinais vitais, dos reflexos protetores e retorno da consciência, prevenção e detecção precoce das complicações.

Para atender aos propósitos citados, a melhor localização dessa sala é próxima ao centro cirúrgico, diminuindo assim a distância a ser percorrida com o paciente, facilitando o acesso das equipes cirúrgicas, quando necessário, respeitando a instabilidade orgânica, imposta pela complexidade da situação, em que o paciente se encontra.

Além da questão da localização, do espaço físico apropriado, um ponto extremamente importante, para que a sala de recuperação anestésica (SRA), como vem sendo chamada desde 1942, atinja sua finalidade e continue a desempenhar sua função é a garantia da especificidade da equipe de profissionais, que deve ser treinada e habilitada para prestar cuidados de alta qualidade e individualizado. (DRAIN; SHIPLEY, 1981).

A individualização dos cuidados prestados na SRA tem sido procurada, não so através de treinamento e orientações básicas da equipe como também pelo assessoramento feito pela monitorização, em desenvolvimento constante

"Professor Assistente do Departamento de Enfermagem Médico Cirúrgica da Escola de Enfermagem da USP 
decorrente do crescente avanço tecnológico, gerando informações sobre funções vitais dos pacientes (FRAULINI, 1987; ARAUJO NETO,1992).

$\mathrm{O}$ que se tem vivenciado, é uma constante busca da individualização do cuidado ao paciente, principalmente nos grandes centros, com maior exatidão e prontidão na deteç̧ão precoce de problemas a que esta exposto. Porém, paralelamente a essa situação ocorre um distanciamento, cada vez maior do profissional de enfermagem, em relação ao cuidado que deve ser oferecido para existência de uma assistência individualizada, específica ao paciente crítico em SRA.

É suposto que existam várias razões para esse desencontro, a escassez de enfermeiros por exemplo, alta rotatividade desses profissionais possam estar associadas. Como cita Utting et al apud NOCITE (1991), os problemas no período de recuperação anestésica resultam da falta de pessoal treinado, capaz de reconhecer sinais de complicações.

Existe para CASTELLANOS (1987) um emaranhado de razões, sobressaindo a indefinição do nosso foco de ação. Para SILVA; FONSECA (1993) outros pontos afetam indiretamente a assistência ao paciente crítico como a falta de estrutura organizacional, ausência de uma filosofia institucional, que priorize o homem.

A informalidade da assistência de enfermagem, prestada sem critérios rígidos de admissão, evolução e alta do paciente em SRA, acarretam um ônus para o paciente e é de grande responsabilidade, não só da equipe mas também da instituição pois existe um número significativo de mortes nesse período em decorrência da falta, nesse setor, da atuação de enfermagem especializada.(NOCITE, 1991).

Como cita ARAUJO NETO (1992) frente a qualquer método, por mais moderno que seja, o monitor ainda mais importante e fidedigno continua sendo a vigilância permanente do profissional em relação ao paciente. Os métodos de monitorização, por mais sofisticados que sejam, devem ser encarados como auxiliares.

Diante dessa situação, fica evidente que a sofisticação oferecida pela monitorização atual, se não encarada como um método auxiliar, não atinge parte de seus propositos, fazendo com que o profissional subestime os riscos decorrentes da doença, do procedimento anestésico cirúrgico, a que se expõe o paciente.

NAIMARK (1990) sugere uma relação de simbiose entre o profissional e a tecnologia, utilizando o melhor de ambos em benefício do paciente.

Diante dessa realidade não se deve descartar, em unidades de cuidados a pacientes críticos, a monitorização feita também pelo registro de parâmetros fisiologicos e avaliação por escores com índices pré-estabelecidos.

A elaboração de um indice por ALDRETE; KROULIK (1970), apresentando no anexo 1 vem embasando a avaliação fisiológica do paciente até nossos dias, devido a aceitabilidade e finalidade a que se propõe, ou seja, sistematizar a observação das condições fisiológicas dos pacientes em SRA de modo simples e objetivo. Mesmo sendo amplamente utilizado questões e sugestões veêm sendo feitas, desde sua criação, relacionadas ás dificuldades encontradas em pontuar isoladamente determinados parâmetros, que avaliam os sistemas comprometidos pelas drogas utilizadas no procedimento anestésico cirúrgico.

A dificudade em encontrar um método não invasivo e que avalia-se a função circulatória por um único sinal, foi expressa pelos autores. A opção feita pela medida indireta da pressão arterial, em decorrência da constância com que é executada, no paciente internado e por ser monitorizada durante todo $o$ ato anestésico

Porém existem algumas variáveis na prática diária, envolvidas com a técnica da medida indireta da pressão arterial que, mesmo com o suporte dado pela avaliação da coloração, devem ser consideradas.

ARCURI (1985); PENICHE (1990) e POUND (1987) em estudos realizados relacionados à medida indireta da pressão arterial questionaram a largura do manguito relacionada à largura do braço, ao seu comprimento levando a hipo ou a hiperestimação dos valores máximos e mínimos da pressão arterial. A ausculta dos sons de Korotkoff feita de modo não padronizado pelos membros da equipe de enfermagem comprometem também a leitura da pressão arterial.

Essas variáveis associadas interferem consequentemente na pontuação dada para o item circulação.

A indefinição do período considerado como préoperatório pelos autores, acarreta dificuldade na escolha do valor da pressão arterial correspondente a esse período. Vale ressaltar que essa medida é tomada como base para o cálculo de variação da pressão arterial permitida para o período pós operatório.

Problemas relacionados e não pontuados são a oligúria, anúria, hemorragia, arritmias que com certeza afetam o sistema cardiovascular.

ALDRE'TE; KROULIK (1970) pontuam de zero a dois $(0-2)$ outros parâmetros rotineiramente observados, e ao final de cada avaliação é efetuada a soma desses escores. Um total de oito (8) a dez (10) pontos indicam que o paciente está em condições de alta da SRA. Essa somatória é atingida pela 
maioria dos pacientes após duas (2) horas de permanência nessa sala. Assim, foram pontuados a atividade muscular, respiração, circulação, consciência e coloração.

Da mesma forma que se questiona a medida da pressão arterial questiona-se também avaliação da atividade muscular. Por exemplo, o paciente com deficit motor, tem a pontuação prejudicada, ao ser avaliado em sua atividade muscular, mesmo tendo conhecimento que esse deficit não é decorrente do procedimento anestésico.

Essa dificuldade era encontrada também quando a coloração era avaliada em pacientes que se apresentam cianóticos por alguma patologia pré existente e receberam a pontuação zero $(0)$, como aquele que apresenta a cianose em decorrência da dificuldade de trocas gasosas provocadas pelos agentes anestésicos ou pela vasoconstrição determinada pela hipotermia. Recentemente substituiu-se a avaliação subjetiva da coloração pela utilização do oxímetro que nos dá a resposta segura sobre a cianose existente

Corroborando com CASTAÑOS (1982)a avaliação da consciência proposta por ALDRETE; KROULIK (1970), causa polêmica entre os membros da equipe, uma vez que a consciência neste período sofre uma oscilação, decorrente da natureza do anestésico. Ficando também prejudicada a averiguação da consciência em pacientes com deficiência mental ou problemas psiquiátricos.

Para CASTAÑOS (1982) deveria se fazer uma avaliação uniforme do grau de recuperação anestésica, quando relacionada a avaliação da consciência sem as divergências existentes. Esse autor propõe uma sistematização, baseada na observação e qualificação, dos sinais dependentes da ação do anestésico sobre o sistema nervoso central, ou seja, avaliação dos reflexos oculares, motilidade, dor, expressão verbal e compreensão de mensagens e ordens simples e não so a condição do paciente estar acordado. Conclui que a pontuação máxima para a consciência avaliada em todos seus subitens citados acima, não significaria completa lucidez. Observou que em pacientes com essa pontuação ainda existia torpor, decorrente do tipo de anestésico utilizado.

A avaliação da função respiratória, como foi sugerida, sem a utilização de aparelhos e testes sofisticados não trouxe dúvidas como os outros itens avaliados. Acredito que a contagem da frequência respiratória e a verificação da expansibilidade toráxica proporcionaram a segurança necessária na avaliação do paciente.

ABRAHÃO; JOAQUIM; MUNESHICA (1990) em estudo feito concluíram que esse índice proposto, por ALDRETE; KROULIK (1970) como único critério de avaliação do paciente não é seguro. Uma pontuação superior a sete (7) não implicaria na diminuição da vigilância, pois nesse período com essa pontuação, foi encontrado maior porcentagem de complicações.

$\mathrm{Na}$ tentativa de melhor avaliar esses pacientes, outros autores também propuseram escores muito semelhantes ao criado em 1970, sempre objetivando a segurança na avaliação e abrangência.

POSSO (1975) com a inclusão da temperatura, pulso, incisão cirúrgica teve como meta diminuir o tempo de permanência dos pacientes na SRA sem por em risco a vida dos mesmos e racionalizar o trabalho do pessoal.

Para FROUST (1985) existem parâmetros universalmente aceitos para avaliar o paciente em SRA, na evolução e alta como a capacidade de respirar profundamente, a presença de reflexos, a estabilidade hemodinâmica, o balanço hídrico estável, a infusão e drenagens funcionantes. De acordo com o autor o tratamento da dor e de problemas em potenciais também devem ser considerados como critérios para a evolução e alta do paciente.

Para SCHNEIDER (1982) podem existir sérias complicações em pacientes mesmo com uma pontuação dez (10) em SRA, segundo o índice proposto por ALDRETE; KROULIK, (1970) decorrentes de problemas não contemplados pelo itens de avaliação. Estabelece então padrões mínimos de alta para o paciente da SRA. Cita que em Cleveland, no hospital universitário, são elaborados critérios que priorizam para alta do paciente, com bloqueio anestésico, a capacidade de reconhecimento da sensação de frio e calor provocada pelos estímulos térmicos além da exigência de movimentação dos membros. Com o acréscimo desses e outros critérios, o autor acredita em melhor qualidade de serviços prestados ao paciente.

Para Mc CONNELL (1980) e GRIFFIN (1989) o estabelecimento de padrões são essenciais para o controle da prática dos profissionais de enfermagem pois, os padrões expressam o conceito que possuem pela qualidade de seu serviço. Sua aplicabilidade consiste em introduzí-los e discutílos com o objetivo de torná-los conhecidos pela equipe de enfermagem. Entendê-los como parte integrante de um processo científico, onde existe a coleta, planejamento, implementação e avaliação, reflete os objetivos da enfermagem em SRA e fornece segurança e individualização ao paciente. Os autores acreditam que os padrões possam ser usados como instrumentos educacionais e de avaliação. Quando auxiliam o profissional recém admitido, guiando-o para o cuidado do paciente desde a sua admissão até a alta da SRA atinge sua função como instrumento educacional e quando o cuidado é 
questionado se correto ou não, o padrão cumpre seu papel de instrumento de avaliação ou auditoria.

Foi com essa preocupação que a Association of Operating Room Nurses (AORN) mostrou como o cuidado deve ser dirigido, determinando um modelo para guiar o desenvolvimento de medidas reais da qualidade de assistência prestada (POBOJEWSKI et al, 1992).

HANNAH (1993) e PIERCE (1990) citam o envolvimento de comissões, associações como a "Joint Commission on Acreditation of Healthcare Organizations" no desenvolvimento de padrões, para avaliação de hospitais, designando um programa formal de educação, preparando a equipe de enfermagem para a população específica a quem prestará o cuidado adequado e com qualidade.

Para WILLS; LINWOOD, (1988) a concretização e a operacionalização da qualidade se dá através de padrões, instrumentos para avaliação, que conceitua como medida ou medidas pelas quais o cuidado de enfermagem pode ser julgado ou comparado. Esses autores defendem padrão como um conjunto de variáveis relacionadas com o nível de desempenho esperado e afirmam que para cada padrão deve existir critérios que são como conceitos relatados das variáveis em questão.

AVELAR et al (1994) com a finalidade de avaliar a qualidade da assistência prestada ao paciente em sala de recuperação anestésica propuseram padrões mínimos de assistência como modelo básico, seguido de critério de avaliação. Obtiveram como resultado da avaliação dos padrões propostos, níveis de alta qualidade para os padrões que dão suporte a assistência, ou seja, aqueles relacionados às condições dos equipamentos e a SRA como local para assistência. Para o padrão que envolve a assistência de enfermagem propriamente dita obtiveram um nível de moderada qualidade. Como conclusão propuseram o estabelecimento de um processo de reflexão contínua para ajustar e adequar as ações de enfermagem aos padrões estabelecidos com a finalidade de atingir um nível de alta qualidade não só aos padrões relacionados ao local e equipamentos mas também para o padrão relacionado a assistência de enfermagem em si.

Como vimos a preocupação com a qualidade de assistência ao paciente tem sido uma constante ainda maior principalmente quando relacionada a alta complexidade que envolve o cuidado do paciente em sala de recuperação anestésica lembrando que esta característica é definida pela subjetividacle do homem .

Acreditando ser necessário uma avaliação segura e eficaz do paciente e lembranclo que nessa fase pós-anestésica as respostas ao trauma anestésico cirúrgico estão em seu ponto máximo de alterações metabólicas e endócrinas (Cheibub, 1991; ABRAHÃO; Joaquim; Muneshica 1990), eu questiono: Como ter abrangência e confiabilidade perante essa complexidade, resguardando a rapidez e prontidão exigidas nesse momento.

Diante desse questionamento fica a sugestão de adicionar aos métodos de avaliação já existentes a construção de pachrões e critérios de avaliação da assistência prestada em sala de recuperação anestésica ao paciente; bem como a validação dos mesmos pois cada SRA precisa desenvolver seus proprios padrões e critérios consonantes com os objetivos da instituição.

Eu acredito já existir os padrões e critérios necessários em uma SRA, porém é preciso formalizálos para garantir a segurança e a qualidade do cuidado, assim como manter a continuidade da assistência de enfermagem, não só entre as unidades cirúrgicas envolvidas, mas principalmente para 0 ser que está sendo cuidado.

\section{REFERÊNCIAS BIBLIOGRÁFICAS}

ABRAHĀO, M.S.M.; Joaquim, E.H.G.; Muneshica, M. Análise crítica da escala de Aldrete - Kroulik como critério de alta na recuperação pós-anestésica. Rev. Bras. Anestesiol., v. 40 p. 172, 1990. Suplement 12 .

Aldrete, J.A.; Kroulik, D. A postanesthetic recovery score. Curr. Rev. Ancstlı., v. 4a, n. 6, p. 924-33, 1970.

Arcuri, E.A.M. Estudo comparativo da medida indireta da pressão arterial com manguito de largura correta e com manguito de largura padrão. Săo Paulo, 1985. 175p. Tese (Doutorado) Instituto de Ciências Biomédicas, Universidade de São Paulo.

Avelar, M.C.Q. et al. Avaliação da qualidade do cuidado de enfermagem em recuperação anestésica. In: CONGRESSO BRASILEIRO DE ENFERMAGEM EM CENTRO CIRÚRGICO, 1, São Paulo, 1993. Anais. São Paulo, Grid Projetos Editoriais, 1994. p.15-27.

CASTAÑOS, C.C. Indices de recuperação. Rev.Bras.Anestesiol., v.32, n.6, p $441-2,1982$.

CASTELLANOS, B.E.P. O trabalho do enfermeiro - a procura e o encontro de um caminho para seu estudo: da abordagem mecanica-funcionalista à pesquisa emancipatória. São Paulo, 1987. 395p. Tese ( Doutorado) - Escola de Enfermagem, Universidade de Sao Paulo.

CHEIBUB, Z.B. Respostas endócrinas à anestesia. Rev. Bras. Anestesiol., v.41, n.5, p.357-63, 1991.

DRAIN, C.B.; SHIPLEY, B.S. Enfermagem na sala de recuperação. Rio de Janeiro, Interamericana, 1981, cap.15, p.241-70: Assistência na sala de recuperação geral.

ELTRINGHAM; R; DURKIN, M; ANDREWS, S. Recuperação pós anestésica. Rio de Janeiro, Colina, 1986. 
FRAULINI, K.E. After anesthesia. A guide for PACU, ICU and medical-surgical nurse. California, Appleton \& Lange. 1987. cap. 1, p. 3-15: Development of the postanesthesia care unit.

FROUST, E.A.M. Why is the patient not a wake yet? In: FROUST, E.A.M. Recovery Room Practice. Blackwell, Scientific Publications, 1985 , cap. 6, p. 87-101.

GRIFFIN, D.N. A tool to develop standards of quality care in pacus. J. Post Anesth. Nurs. v.4, n.2, p.99-102, 1989.

HANNAH, B.A. Establishing clinical competence in postanesthesia care nursing. J. of Post Anestl. Nurs. v. 8, n. 3, p. 187-92, 1993.

McCONNELL E.A. Aplying recovery room standards. AORN J., v. 31, n. 5 , p. $796-98,1980$.

NAIMARK, T.M. A comparasion study of the post anesthesia recovery score and pulse oximetry. J. Post Anesth. Nurs., v. 5, n. 3, p. $168-76,1990$.

ARAUJO NETO, J.P. de. Monitorização em Anestesia. Rev. Bras. Anestesiol., v. 42, n. 2, p. 1, 1992.

NOCITE, J.R. Recuperação pós anestésica: aspectos gerais. Rev. Bras. Anestesiol., v.37, n.3, p.161-7, 1987.

NOCITE, J.R. Mortalidade associada à anestesia: estudos epidemiológicos. Rev. Bras. Anestesiol., v.41, n.1, p.3-7, 1991.
PENICHE, A.C.G. Medida indireta da pressão arterial em função da largura do manguito em pacientes $n$ as fases pré-operatória imediata, recepção no centro cirúrgico e pós-anestésica. São Paulo, 1990. 42p. Dissertação (Mestrado) - Escola de Enfermagem, Universidade de São Paulo.

PIERCE, E.C. Anesthesia risk modification: reducing the incidence of mishaps. Curr. Rev. Anestl.. Care Nurses. v.12, n.2, p.9-16, 1990.

POBOJEWSKI, B.J. et al. Documenting nursing process in the perioperative setting. AORN J., v.56, n.1, p.98-112, 1992.

POSSO, M.B.S. Avaliação das condições dos pacientes em sala de recuperação anestésica. Rev.Esc.Enf.USP, v.9, n.3, p.9-23, 1975 .

POUND, J.L. Consistency in measurement of blood pressure in the postanesthesia care unit. J. Post Anesth. Nurs., v.2, n.2, p. $78-83,1987$.

SCHNEIDER, M. Meeting the criteria for discharge. Curr. rev. recovery room nurs., v.4, n.6, p.41-8, 1982.

SILVA, F.M.; FONSECA, M.M. Motivos que levam as enfermeiras de centro cirúrgico a não realizarem uma assistência de enfermagem de forma sistematizada. In: CONGRESSO BRASILEIRO DE ENFERMAGEM EM CENTRO CIRÚRgICO,1, Sao Paulo, 1993. Anais São Paulo, Grid Projetos Editoriais, 1994. p.101-5.

WILLS, L.D.: LINWOOD, M.E. Measuring the quality of care. J. Nurs. Qual. Assur., v.2, n.2, p.15-6, 1988. 


\section{ANEXO 1}

\section{ÍNDICE DE RECUPERAÇÃO PÓS ANESTÉSICA} ALDRETE E KROULIK

ATIVIDADE

[2] Apto a mover 4 extremidades

[1] Apto a mover 2 extremidades

[0] Apto a mover 0 extremidades

RESPIRAÇÃO

[2] Apto respirar profundo e tossir

[1] Dispnéia ou respiração limitada

[0] Apnéia

CIRCULAÇÃO

[2] PA $+20 \%$ valor pré-anestésico

[1] PA $+25 \%$ valor pré-anestésico

[0] PA + 50\% valor pré-anestésico

CONSCIÊNCIA

[2] Desperto totalmente

[1] Desperto ao chamar

[0] Não responde estímulos verbais

COLORAÇÃO

[2] Róseo

[1] Pálido, marmóreo, ictérico

[0] Cianótico 